\section{PL04.2 WHERE ARE THE MEN? STI AND SEXUAL HEALTH SERVICES FOR MEN}

Ravi Verma. Regional Director and Madhumita Das, International Centre for Research on Women, New Delhi, India

\subsection{6/sextrans-2015-052270.11}

This presentation describes why men are invisible and do not seek sexual health services, mainly STI care and treatment. The review examines ${ }^{1}$ (i) how masculinity promotes STI/HIV infections; (ii) why men avoid seeking care and services and (ii) what are the means and ways through which services can reach men. The analysis attempts to answer the pathways of how social norms influence roles, behaviours and expectations and reinforce conditions on men through masculinity norms. In addition, it also describes structural barriers such as predominant 'Feminised' clinic spaces that inhibit men to seek services and adopt preventive behaviours.

Culture and social norms influence men's and women's risk through gender roles and relationships and ultimately determine whether, with whom and how various preventive technologies and sexual health services may or may not be used. Dominant masculinity norms and restrictive legal environments preclude men to reveal their sexual identities and preferences creating vulnerabilities to both men and women. Men's sexual health practices have been linked to diverse enactments of masculinities that can both negatively and positively influence men's wellbeing. Hegemonic masculinity idealises men's bodies as robust and resilient (Charles and Walters 2008) and are more amenable to self-management than to seeking help from healthcare providers. Many men who engage in sex with other men are married in countries like India. Men's perception of risk from such behaviours is also often low - especially if one is the "penetrator". Men who engaged in extramarital sex (whether with other women or men) were six times more likely to report wife abuse than those who did not. Yet, gender norms perpetuate women's submission to coercive sex in marriage and prevent frank discussions about sexuality and risk. While the introduction of microbicides may act as a catalyst, more encompassing gender transformative strategies were needed to reduce men's and women's risk of HIV and STI.

The review also opens up the ways of finding an alternative means which can open up the possible route for them to utilise the services.

\section{REFERENCE}

1 Charles N, Walters V. 'Men are leavers alone and women are worriers': gender differences in discourses of health. Health Risk Society 2008;10:117-132

\section{PL04.3 SEXUALLY TRANSMITTED INFECTIONS IN MEN WHO HAVE SEX WITH MEN}

Henry JC de Vries. Professor of Skin Infections, STI Outpatient Clinic, Public Health Service Amsterdam, Department of Dermatology, and CINIMA, Academic Medical Center, University of Amsterdam, and Centre for Infectious Diseases Control, National Institute for Public Health and the Environment (CIb/RIVM), Bilthoven, The Netherlands

\subsection{6/sextrans-2015-052270.12}

Homosexuality is a global human phenomenon that can either describe the behaviour, preference or identity in relation to sexuality with someone from the same sex. Although homosexuality is becoming more and more accepted in many countries of the world, in large parts of society it is still considered as deviant, unnatural and to be discouraged. A considerable proportion of countries in Africa, the Caribbean and the Middle East have some form of criminal laws against homosexual behaviour. Criminalisation of and atrocities towards homosexuals seriously hinders access to health care and endanger not only minority groups, but in the long run the population at large. The American Psychiatric Association removed homosexuality from its list of disorders over 35 years ago, yet homophobia among physicians is still widely prevalent. From a historical point of few, men who have Sex with Men (MSM) form a relatively new epidemiological risk group for STI. Yet it was the deadly HIV/AIDS epidemic that ignited a strong gay activist movement in the United States and Europe fighting for more research to find a cure and diminish disease transmission among MSM. Phylogenetic techniques and subsequent cluster analysis of STI pathogens have recently opened the possibility to unravel STI transmission networks in more detail and increased the insight into transmission mechanisms in MSM. To perform correct management, clinicians evaluating men with male-male sex contacts for STI-related complaints or STI screening must obtain a thorough sexual history. Emerging STI like lymphogranuloma venereum, hepatitis $\mathrm{C}$ and multidrug resistant Neisseria gonorrhoeae strains have been described first in MSM. STI-related proctitis often occur in MSM. Within the MSM population, HIV positive patients form a special group affected by STI-related diseases like anal carcinoma and neurosyphilis. The final part of this talk concludes with recommendations to reduce the STI burden in MSM.

\section{Plenary Session PL05 \\ Wednesday 16 September 2015}

\subsection{0am - 10.45am}

\section{PL05.1 TREATMENT TO PREVENT HIV: DOES TIMING MATTER?}

Myron S Cohen. Associate Vice Chancellor for Global Health, Yeargan-Bate Distinguished Professor of Medicine, Microbiology and Immunology, University of North Carolina at Chapel Hill, USA

\subsection{6/sextrans-2015-052270.13}

The horizontal transmission of HIV infection depends primarily on the concentration of virus in the blood and genital secretions. Accordingly, sexual transmission of HIV can be virtually eliminated with successful antiretroviral treatment (ART); not surprisingly, vulnerability for an HIV negative partner exists early in treatment and when/if treatment fails. Treatment of HIV (even at high CD4 count) improves health and prevents neoplasms and tuberculosis. These results have led to widespread recommendation for universal testing and treatment (UTT), limited only by infrastructure and not stage of disease. However, the detection and treatment of acute and early infection remains a challenge. There is strong evidence that treatment of acute and early HIV has both health and public health benefit. And people treated during acute infection have a smaller viral reservoir ("latent pool") and may be better candidates for attempts at viral eradication (i.e. cure). However, detection of patients with very early infection remains a challenge. Point of care tests currently available to detect acute infection do not perform well. Syndromic algorithms for detection of acute infection are available but they have not been widely used, even in Africa. Use of viral 